\title{
Hypermonogenic Plane Wave Solutions of the Dirac equation in Superspace
}

\author{
Alí Guzmán Adán ${ }^{1}$, Heikki Orelma², Frank Sommen ${ }^{1}$ \\ ${ }^{1}$ Clifford Research Group, Department of Mathematical Analysis, Faculty of Engineering and \\ Architecture, Ghent University, Krijgslaan 281, 9000 Gent, Belgium. \\ ${ }^{2}$ Laboratory of Civil Engineering, Tampere University, FI-33014 Tampere University, Finland. \\ Ali.GuzmanAdan@UGent.be, Heikki.Orelma@tuni.fi, Franciscus.Sommen@UGent.be
}

\begin{abstract}
In this paper, we obtain Cauchy-Kovalevskaya theorems for hypermonogenic superfunctions depending only on purely bosonic and fermionic vector variables. In addition, we use these results to construct plane wave examples of such functions.
\end{abstract}

Mathematics Subject Classification (2000). 30G35, 30A05, 58C50

Keywords. Clifford analysis, superspace, hypermonogenic functions, plane waves, CauchyKovalevskaya extension

\section{Introduction}

Supermanifolds and in particular superspaces play an important rôle in contemporary theoretical physics, e.g. in the particle theory of supersymmetry, supergravity or superstring theories, etc. Superspaces are equipped with both a set of commuting co-ordinates and a set of anti-commuting co-ordinates. From the mathematical point of view, they have been studied using algebraic and geometrical methods (see e.g. [2, 16, 24]). More recently, harmonic and Clifford analysis have been extended to superspace by introducing some important differential operators (such as Dirac and Laplace operators) and by studying special functions and orthogonal polynomials related to these operators, see e.g. [6, 8, 11, 23].

Clifford analysis in $\mathbb{R}^{m}$ is a function theory for null-solutions of the Dirac operator $\partial_{x}=$ $\sum_{j=1}^{m} e_{j} \partial_{x_{j}}$, where $\left\{e_{1}, \ldots, e_{m}\right\}$ generates the Clifford algebra $\mathbb{R}_{m}$ by the defining relations $e_{i} \bar{e}_{j}+$ $e_{j} e_{i}=-2 \delta_{i j}$. Standard references on the classical Clifford setting are [3, 15, 20]. Among the different features of Clifford analysis one finds the theory of so-called hypermonogenic functions introduced in the beginning of the 90's by Heinz Leutwiler and Sirkka-Liisa Eriksson, see e.g. $[17,25]$. Hypermonogenic functions are null-solutions of the modified Dirac operator

$$
M=e \partial_{r}+\partial_{\underline{y}}+\frac{p-1}{r} e \cdot
$$

where $p$ is a natural number and $e$ denotes the interior multiplication, i.e.

$$
e \cdot a_{k}=\frac{1}{2}\left(e a_{k}-(-1)^{k} a_{k} e\right),
$$

for any $k$-vector $a_{k} \in \mathbb{R}_{m}$, see [15]. The extra generator $e$ gives rise to the Clifford algebra $\mathbb{R}_{m+1}$ by the defining relations $e_{i} e_{j}+e_{j} e_{i}=-2 \delta_{i j}, e^{2}=-1$ and $e e_{j}=-e_{j} e$. The modified Dirac 
operator $M$ acts on $\mathbb{R}_{m+1^{-}}$-valued functions defined on an open subset of the upper half-space $\mathbb{R}_{+}^{m+1}=\left\{(r, \underline{y}) \in \mathbb{R} \times \mathbb{R}^{m}: r>0\right\}$.

The resulting hypermonogenic function theory is related to a hyperbolic upper half-space model. The theory has its own advantages, e.g. paravector power functions may be included in the kernel of the operator. The reader may find more information and recent research results for example in $[17,18,19,25]$.

In the recent paper [22], we have deduced a correspondence between the set of the nullsolutions of the modified Dirac operator $M$ and the null-solutions of the biaxial Dirac operator $\partial_{\underline{x}}+\partial_{\underline{y}}$ where the pair of vector variables $(\underline{x}, \underline{y})$ is defined in $\mathbb{R}^{p} \times \mathbb{R}^{q}$. In particular, it was proven that functions of the form $f=A+e B=\sum_{j=0}^{\infty} \underline{x}^{j} f_{j}(\underline{y})$ satisfy

$$
\partial_{\underline{x}}(A+e B)+\partial_{\underline{y}}(A+e B)=e \partial_{r}(A+e B)+\partial_{\underline{y}}(A+e B)+\frac{p-1}{r} e \cdot(A+e B),
$$

whereby we consider Clifford algebra valued functions $A, B$ that are radial with respect to $\underline{x} \in \mathbb{R}^{p}$, and we put $r=|\underline{x}|$ and $e=\frac{x}{r}$.

This last approach to hypermonogenic functions only depends on two orthogonal vector variables $\underline{x}$ and $y$. This makes it suitable for an extension to superanalysis where the notions of vector variable and Dirac operator incorporate the use of fermionic (i.e. anti-commuting) variables. In this paper, we study three particular cases of hypermonogenic superfunctions depending on two supervector variables $\mathbf{x}$ and $\mathbf{y}$ where at least one of them is purely fermionic, i.e. it has negative dimension. In particular, we study functions of the forms:

- $F(\underline{\grave{x}}, \underline{y})=\sum_{j=0}^{\infty} \underline{x}^{j} F_{j}(\underline{y})$

- $F(\underline{x}, \underline{y})=\sum_{j=0}^{\infty} \underline{x}^{j} F_{j}(\underline{y})$

- $F(\underline{x}, \underline{y})=\sum_{j=0}^{\infty} \underline{x}^{j} F_{j}(\underline{y})$

where $\underline{x}, \underline{y}$ are bosonic vector variables and $\underline{x}, \underline{y}$ are fermionic vector variables. These cases show important differences and new insights with respect to the classical setting where only purely bosonic vector variables are considered.

The structure of the paper is as follows. We start in Section 2 with some preliminaries on the Clifford function theory in the superspace setting. In Section 3, we study hypermonogenic functions of the aforementioned forms by determining Cauchy-Kovalevskaya type extensions for each case. Finally, in Section 4 we apply these results in obtaining concrete examples of hypermonogenic superfunctions. In particular, we focus our attention to hypermonogenic solutions of plane wave type. In the standard theory of monogenic functions, plane waves play a crucial rôle in connection with Fourier analysis. Moreover it is possible to represent general solutions of the Dirac operator as linear combinations of two-dimensional monogenic functions (plane waves).

\section{Preliminaries}

In this paper, we follow the usual approach of Clifford analysis in superspace which considers $m$ commuting variables $x_{1}, \ldots, x_{m}$ and $2 n$ anti-commuting variables $\grave{x_{1}}, \ldots, \grave{x_{2 n}}$ in a purely symbolic way, i.e.

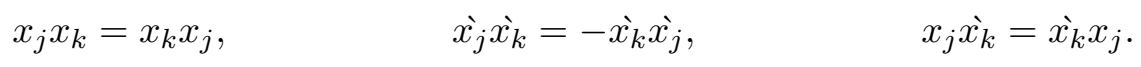


The variables $x_{1}, \ldots, x_{m}$ are thus generators of the polynomial algebra $\mathbb{R}\left[x_{1}, \ldots, x_{m}\right]$ while $\grave{x_{1}}, \ldots, \grave{x_{2 n}}$ generate a Grassmann algebra $\mathfrak{G}_{2 n}$. We will denote by $\mathfrak{G}_{2 n}^{(e v)}$ and $\mathfrak{G}_{2 n}^{(\text {odd })}$ the subalgebras of even and odd elements of $\mathfrak{G}_{2 n}$ respectively. The algebra of super-polynomials, i.e. polynomials in the variables $x_{1}, \ldots, x_{m}, \grave{x_{1}}, \ldots, \grave{x_{2 n}}$, is defined by

$$
\mathcal{P}:=\operatorname{Alg}_{\mathbb{R}}\left(x_{1}, \ldots, x_{m}, \grave{x_{1}}, \ldots, \grave{x_{2 n}}\right)=\mathbb{R}\left[x_{1}, \ldots, x_{m}\right] \otimes \mathfrak{G}_{2 n} .
$$

The bosonic and fermionic partial derivatives $\partial_{x_{j}}, \partial_{\grave{x_{j}}}$ are defined as endomorphisms on $\mathcal{P}$ by the relations

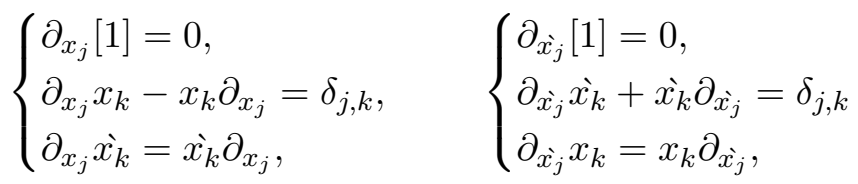

that can be applied recursively. From this definition it immediately follows that $\partial_{x_{j}} \partial_{x_{k}}=\partial_{x_{k}} \partial_{x_{j}}$, $\partial_{\breve{x_{j}}} \partial_{\grave{x_{k}}}=-\partial_{\grave{x_{k}}} \partial_{\grave{x_{j}}}$ and $\partial_{x_{j}} \partial_{\grave{x_{k}}}=\partial_{\grave{x_{k}}} \partial_{x_{j}}$.

The flat supermanifold corresponding to the variables $x_{j}, \grave{x_{j}}$ is denoted by $\mathbb{R}^{m \mid 2 n}$. The full algebra of functions on this supermanifold is $C^{\infty}\left(\mathbb{R}^{m}\right) \otimes \mathfrak{G}_{2 n}$ where $C^{\infty}\left(\mathbb{R}^{m}\right)$ denotes the space of infinitely many differentiable complex-valued functions defined in $\mathbb{R}^{m}$. The partial derivatives $\partial_{x_{j}}, \partial_{\grave{x}_{j}}$ extend from $\mathcal{P}$ to $C^{\infty}\left(\mathbb{R}^{m}\right) \otimes \mathfrak{G}_{2 n}$ by density.

The super Clifford algebra $\mathcal{C}_{m, 2 n}$ is generated by $m$ orthogonal Clifford generators $e_{1}, \ldots, e_{m}$ and $2 n$ symplectic Clifford generators $\grave{e}_{1}, \ldots, \grave{e_{2 n}}$ which are subjected to the multiplication rules

$$
e_{j} e_{k}+e_{k} e_{j}=-2 \delta_{j, k}, \quad e_{j} \grave{e_{k}}+\grave{e_{k}} e_{j}=0, \quad \grave{e_{j}} \grave{e}_{k}-\grave{e_{k}} \grave{e_{j}}=g_{j, k},
$$

where $g_{j, k}$ is a symplectic form defined by

$$
g_{2 j, 2 k}=g_{2 j-1,2 k-1}=0, \quad g_{2 j-1,2 k}=-g_{2 k, 2 j-1}=\delta_{j, k}, \quad j, k=1, \ldots, n .
$$

These generators are combined with the algebra of super-polynomials $\mathcal{P}$ giving rise to the algebra of Clifford valued super-polynomials $\mathcal{P} \otimes \mathcal{C}_{m, 2 n}$; where elements in

$$
\mathcal{C}_{m, 2 n}:=\operatorname{Alg}_{\mathbb{R}}\left(e_{1}, \ldots, e_{m}, \grave{e_{1}}, \ldots, \grave{e_{2 n}}\right)
$$

commute with elements in $\mathcal{P}$. Also the partial derivatives $\partial_{x_{j}}, \partial_{\grave{x}_{j}}$ commute with the elements in the algebra $\mathcal{C}_{m, 2 n}$.

The most important element of the algebra $\mathcal{P} \otimes \mathcal{C}_{m, 2 n}$ is the supervector variable

$$
\mathbf{x}=\underline{x}+\underline{\grave{x}}=\sum_{j=1}^{m} x_{j} e_{j}+\sum_{j=1}^{2 n} \grave{x_{j}} \grave{e}_{j} .
$$

where $\underline{x}=\sum_{j=1}^{m} x_{j} e_{j}$ and $\underline{\grave{x}}=\sum_{j=1}^{2 n} \grave{x_{j}} \grave{e_{j}}$ are its bosonic and fermionic projections respectively. While the bosonic projection $\underline{x}=\sum_{j=1}^{m} x_{j} e_{j}$ constitutes an usual Clifford vector variable, the fermionic vector variable $\underline{\grave{x}}=\sum_{j=1}^{2 n} \grave{x_{j}} \grave{e}_{j}$ brings new insights. For example, $\underline{\grave{x}}$ is nilpotent. Indeed,

$$
\underline{x}^{2 n}=n ! \grave{x_{1}}{\grave{x_{2}}}^{2} \cdot \grave{x}_{2 n-1}{\grave{x_{2 n}}},
$$

which means that $\underline{x}^{2 n+1}=0$.

Supervector variables define a representation of the so-called radial algebra in the superspace framework. To link radial algebra with superanalysis it suffices to verify that the anti-commutator 
of every pair of vector variables is a central element (see e.g. [6, 7]). The anti-commutator $\{\mathbf{x}, \mathbf{y}\}:=\mathbf{x y}+\mathbf{y} \mathbf{x}$ allows to define the inner product of the two supervectors $\mathbf{x}$ and $\mathbf{y}$ as follows

$$
\langle\mathbf{x}, \mathbf{y}\rangle=-\frac{1}{2}\{\mathbf{x}, \mathbf{y}\}=\sum_{j=1}^{m} x_{j} y_{j}-\frac{1}{2} \sum_{j=1}^{n}\left(\grave{x}_{2 j-1} \grave{y}_{2 j}-\grave{x}_{2 j} \grave{y}_{2 j-1}\right) .
$$

Functions in $C^{\infty}\left(\mathbb{R}^{m}\right) \otimes \mathfrak{G}_{2 n}$ can be explicitly written as

$$
F(\mathbf{x})=F(\underline{x}, \underline{x})=\sum_{A \subset\{1, \ldots, 2 n\}} F_{A}(\underline{x}) \underline{x}_{A},
$$

where $F_{A}(\underline{x}) \in C^{\infty}\left(\mathbb{R}^{m}\right)$ and $\underline{\grave{x}}_{A}$ is defined as $\grave{\dot{x}_{1}} \ldots \grave{x_{j_{k}}}$ with $A=\left\{j_{1}, \ldots, j_{k}\right\}, 1 \leq j_{1}<\ldots<$ $j_{k} \leq 2 n$. Similarly, one may consider other spaces of superfunctions of the form $\mathcal{F} \otimes \mathfrak{G}_{2 n}$ where $\mathcal{F}=C^{k}(\Omega), L_{2}(\Omega), \ldots$, with $\Omega \subset \mathbb{R}^{m}$. Every superfunction can be written as the sum $F(\mathbf{x})=F_{0}(\underline{x})+\mathbf{F}(\underline{x}, \underline{x})$ where the complex-valued function $F_{0}(\underline{x})=F_{\emptyset}(\underline{x})$ is called the body $F$, and $\mathbf{F}=\sum_{|A| \geq 1} F_{A}(\underline{x}) \underline{x}_{A}$ is the nilpotent part of $F$. Indeed, it is clearly seen that $\mathbf{F}^{2 n+1}=0$. When considering the aforementioned Clifford generators, this space of superfunctions extends to the space of Clifford-valued superfunctions $C^{\infty}(\Omega) \otimes \mathfrak{G}_{2 n} \otimes \mathcal{C}_{m \mid 2 n}$.

It is possible to produce interesting even superfunctions out of known functions from classical analysis. Indeed, consider an analytic function $F: \mathbb{C} \rightarrow \mathbb{C}$ and an even superfunction $a=a_{0}+\mathbf{a} \in$ $C^{\infty}\left(\mathbb{R}^{m}\right) \otimes \mathfrak{G}_{2 n}^{(e v)}$ where $a_{0}$ and $\mathbf{a}$ are the body and nilpotent part of $a$ respectively. Then the superfunction $F(a(\mathbf{x})) \in C^{\infty}\left(\mathbb{R}^{m}\right) \otimes \mathfrak{G}_{2 n}^{(e v)}$ is defined, through the Taylor expansion of $F$, as

$$
F(a)=F\left(a_{0}+\mathbf{a}\right)=\sum_{j=0}^{n} \frac{\mathbf{a}^{j}}{j !} F^{(j)}\left(a_{0}\right),
$$

where $F^{(j)}$ denotes the $j$-th derivative of the analytic complex function $F$.

The bosonic and fermionic Dirac operators are defined by

$$
\partial_{\underline{x}}=\sum_{j=1}^{m} e_{j} \partial_{x_{j}}, \quad \partial_{\underline{\grave{x}}}=2 \sum_{j=1}^{n}\left(\grave{e_{2 j}} \partial_{\grave{x_{2 j-1}}}-\grave{e_{2 j-1}} \partial_{\grave{x_{2 j}}}\right),
$$

which lead to the left and right super Dirac operators (super-gradient) $\partial_{\mathbf{x}} \cdot=\partial_{\underline{x}} \cdot-\partial_{\underline{x}} \cdot$ and $\cdot \partial_{\mathbf{x}}=-\cdot \partial_{\underline{x}}-\cdot \partial_{\underline{x}}$ respectively. As in the classical Clifford setting, the action of $\partial_{\mathbf{x}}$ on the vector variable $\mathbf{x}$ results in the superdimension

$$
M:=\partial_{\mathbf{x}}[\mathbf{x}]=[\mathbf{x}] \partial_{\mathbf{x}}=\partial_{\underline{x}}[\underline{x}]-\partial_{\underline{x}}[\underline{x}]=m-2 n .
$$

The action of the super Dirac operator on any positive power of $\mathbf{x}$ is given by

$$
\partial_{\mathbf{x}}\left[\mathbf{x}^{j}\right]=c(M, j) \mathbf{x}^{j-1}, \quad \text { where } \quad c(M, j)= \begin{cases}j, & \text { if } j \text { is even, } \\ M+j-1, & \text { if } j \text { is odd } .\end{cases}
$$

Given an open set $\Omega \subset \mathbb{R}^{m}$, a function $F \in C^{1}(\Omega) \otimes \mathfrak{G}_{2 n} \otimes \mathcal{C}_{m, 2 n}$ is said to be (left) super monogenic if $\partial_{\mathbf{x}}[F]=0$. As in the purely bosonic case, the super Dirac operator factorizes the super Laplace operator, i.e.

$$
\begin{aligned}
& \Delta_{\underline{x}}=-\partial_{\underline{x}}^{2}=\sum_{j=1}^{m} \partial_{x_{j}}^{2}, \\
& \Delta_{\mathbf{x}}=-\partial_{\mathbf{x}}^{2}=\Delta_{\underline{x}}+\Delta_{\underline{x}}=\sum_{j=1}^{m} \partial_{x_{j}}^{2}-4 \sum_{j=1}^{n} \partial_{\grave{x}_{2 j-1}} \partial_{\grave{x}_{2 j}} .
\end{aligned}
$$


Hence monogenicity still constitutes a refinement of harmonicity in superanalysis. More details on the theory of super-monogenic and super-harmonic functions can be found for instance in $[4,5,8,9,10,21]$.

\section{Hypermonogenic functions in superspace}

In this section, we will introduce the notion of hypermonogenic function in superspace and study some important particular cases. In general, we consider functions of the two independent supervector variables

$$
\mathbf{x}=\underline{x}+\underline{\grave{x}}=\sum_{j=1}^{p} x_{j} e_{j}+\sum_{j=1}^{2 r} \grave{x_{j}} \grave{e_{j}} \quad \text { and } \quad \mathbf{y}=\underline{y}+\underline{y}=\sum_{j=1}^{q} y_{j} e_{p+j}+\sum_{j=1}^{2 s} \grave{y_{j}} \grave{e_{2} r+j},
$$

where the elements $e_{1}, \ldots, e_{p}, e_{p+1}, \ldots, e_{p+q}, \grave{e_{1}}, \ldots, \grave{e_{2 r}}, \grave{e_{2 r+1}}, \ldots, \grave{e_{2 r+2 s}}$ generate the super Clifford algebra $\mathcal{C}_{m, 2 n}$ with $m=p+q$ and $n=r+s$. The superdimensions of the vector variables $\mathbf{x}$ and $\mathbf{y}$ are denoted by $P:=p-2 r$ and $Q:=q-2 s$ respectively. Thus the supervector $\mathbf{x}+\mathbf{y}$ has superdimension $M=P+Q$ and satisfy that $(\mathbf{x}+\mathbf{y})^{2}=\mathbf{x}^{2}+\mathbf{y}^{2}$ since $\mathbf{x}$ and $\mathbf{y}$ are orthogonal, i.e. $\mathbf{x y}=-\mathbf{y x}$.

Definition 1. A differentiable superfunction $F(\mathbf{x}, \mathbf{y})$ is said to be hypermonogenic if it is of the form

$$
F(\mathbf{x}, \mathbf{y})=\sum_{j=0}^{\infty} \mathbf{x}^{j} F_{j}(\mathbf{y})
$$

and satisfy $\left(\partial_{\mathbf{x}}+\partial_{\mathbf{y}}\right) F(\mathbf{x}, \mathbf{y})=0$.

Let us now study some important particular cases of hypermonogenic superfunctions.

\subsection{Case $\mathrm{x}=\underline{\grave{x}}$.}

In this case, $\mathrm{x}=\underline{x}$ is a purely fermionic vector variable and hence nilpotent. Then the expansion (8) of the superfunction $F$ has a finite number of terms, i.e.

$$
F(\underline{\grave{x}}, \mathbf{y})=\sum_{j=0}^{2 r} \underline{x}^{j} F_{j}(\mathbf{y})
$$

Proposition 1. A superfunction $F(\underline{\grave{x}}, \mathbf{y})$ of the form (8) is hypermonogenic if and only if

$$
\left\{\begin{array}{l}
\partial_{\mathbf{y}} F_{2 r}=0 \\
c(-2 r, j+1) F_{j+1}=(-1)^{j+1} \partial_{\mathbf{y}} F_{j}, \quad j=2 r-1, \ldots, 0,
\end{array}\right.
$$

where $c(-2 r, j+1)$ is defined in $(7)$.

Proof. It is easily seen that $\partial_{\mathbf{y}} \underline{x}^{j}=(-1)^{j} \underline{x}^{j} \partial_{\mathbf{y}}$. Thus

$$
\begin{aligned}
\left(\partial_{\underline{x}}+\partial_{\mathbf{y}}\right) F & =\sum_{j=0}^{2 r}\left(\partial_{\underline{x}}+\partial_{\mathbf{y}}\right)\left[\underline{x}^{j} F_{j}\right]=\sum_{j=0}^{2 r} \partial_{\underline{x}}\left[\underline{x}^{j}\right] F_{j}+(-1)^{j} \underline{x}^{j} \partial_{\mathbf{y}} F_{j} \\
& =\sum_{j=1}^{2 r} c(-2 r, j) \underline{x}^{j-1} F_{j}+\sum_{j=0}^{2 r}(-1)^{j} \underline{x}^{j} \partial_{\mathbf{y}} F_{j} \\
& =\sum_{j=0}^{2 r-1} \underline{x}^{j}\left(c(-2 r, j+1) F_{j+1}+(-1)^{j} \partial_{\mathbf{y}} F_{j}\right)+\underline{x}^{2 r} \partial_{\mathbf{y}} F_{2 r} .
\end{aligned}
$$


The previous proposition shows a way of constructing hypermonogenic superfunctions in this case. This construction starts with a monogenic function $F_{2 r}(\mathbf{y})$ and follows by successively finding (up to some real constants) primitive functions $F_{2 r-1}, F_{2 r-2}, \ldots, F_{0}$.

It is also possible to construct hypermonogenic superfunctions in this case taking $F_{0}$ as initial function. Indeed, the recursion formulae (9) can be rewritten as

$$
\begin{array}{rlrl}
F_{2 j} & =\frac{(r-j) !}{2^{2 j} j ! r !} \partial_{\mathbf{y}}^{2 j} F_{0}, & & 0 \leq j \leq r, \\
F_{2 j+1} & =\frac{(r-j-1) !}{2^{2 j+1} j ! r !} \partial_{\mathbf{y}}^{2 j+1} F_{0}, & 0 \leq j \leq r-1,
\end{array}
$$

where $F_{0}$ is such that $\partial_{\mathbf{y}}^{2 r+1} F_{0}=0$. Thus Proposition 1 can be written in the following terms.

Corollary 1. A superfunction $F(\underline{\dot{x}}, \mathbf{y})$ of the form (8) is hypermonogenic if and only if

$$
F(\underline{\grave{x}}, \mathbf{y})=\frac{1}{r !}\left(\sum_{j=0}^{r} \frac{(r-j) !}{j !} \frac{\underline{x}^{2 j} \partial_{\mathbf{y}}^{2 j}}{2^{2 j}}+\sum_{j=0}^{r-1} \frac{(r-j-1) !}{j !} \frac{\underline{x}^{2 j+1} \partial_{\mathbf{y}}^{2 j+1}}{2^{2 j+1}}\right)\left[F_{0}\right]
$$

and $\partial_{\mathbf{y}}^{2 r+1} F_{0}=0$

\subsection{Case $\mathrm{x}=\underline{x}$.}

This case is very similar to the purely bosonic setting treated in [15, Sect. 5.1]. Indeed, the expansion of the superfunction $F(\underline{x}, \mathbf{y})$ has the form

$$
F(\underline{x}, \mathbf{y})=\sum_{j=0}^{\infty} \underline{x}^{j} F_{j}(\mathbf{y})
$$

and therefore,

$$
\left(\partial_{\mathbf{x}}+\partial_{\mathbf{y}}\right) F=\sum_{j=0}^{\infty}\left(-\partial_{\underline{x}}+\partial_{\mathbf{y}}\right)\left[\underline{x}^{j} F_{j}\right]=\sum_{j=0}^{\infty} \underline{x}^{j}\left(c(p, j+1) F_{j+1}+(-1)^{j} \partial_{\mathbf{y}} F_{j}\right) .
$$

Then $F(\underline{x}, \mathbf{y})$ is hypermonogenic if and only if the following recurrence formula holds

$$
F_{j+1}=\frac{(-1)^{j+1}}{c(p, j+1)} \partial_{\mathbf{y}} F_{j}, \quad j=0,1, \ldots
$$

This means that the functions $F_{j}$ are uniquely determined by the formulae

$$
F_{2 j}=\frac{(-1)^{j}}{2^{2 j} j !} \frac{\Gamma\left(\frac{p}{2}\right)}{\Gamma\left(\frac{p}{2}+j\right)} \partial_{\mathbf{y}}^{2 j} F_{0} \quad \text { and } \quad F_{2 j+1}=\frac{(-1)^{j+1}}{2^{2 j+1} j !} \frac{\Gamma\left(\frac{p}{2}\right)}{\Gamma\left(\frac{p}{2}+j+1\right)} \partial_{\mathbf{y}}^{2 j+1} F_{0} .
$$

Writing $\partial_{\mathbf{y}}^{2}=-\Delta_{\mathbf{y}}$ and $\underline{x}^{2}=-|\underline{x}|^{2}$ one obtains the following result, see Theorem 5.1.1 in [15].

Proposition 2. A superfunction $F(\underline{x}, \mathbf{y})$ of the form (8) is hypermonogenic if and only if

$$
F(\underline{x}, \mathbf{y})=\Gamma\left(\frac{p}{2}\right)\left(\frac{|\underline{x}| \sqrt{\Delta_{\mathbf{y}}}}{2}\right)^{-\frac{p}{2}}\left(\frac{|\underline{x}| \sqrt{\Delta_{\mathbf{y}}}}{2} J_{\frac{p}{2}-1}\left(|\underline{x}| \sqrt{\Delta_{\mathbf{y}}}\right)-\frac{\underline{x} \partial_{\mathbf{y}}}{2} J_{\frac{p}{2}}\left(|\underline{x}| \sqrt{\Delta_{\mathbf{y}}}\right)\right)\left[F_{0}\right]
$$

where $J_{\nu}(z)=\frac{z^{\nu}}{2^{\nu}} \sum_{j=0}^{\infty} \frac{(-1)^{j} z^{2 j}}{2^{2 j} j ! \Gamma(\nu+j+1)}$ is the Bessel function of the first kind of order $\nu$ and $\sqrt{\Delta_{\mathbf{y}}}$ denotes the square root of the super laplacian $\Delta_{\mathbf{y}}$ (of which only even powers occur in the resulting series). 


\section{Construction of hypermonogenic plane waves in superspace}

In this section, we construct some examples of hypermonogenic superfunctions of plane wave type in the aforementioned particular cases. In general, plane waves are of the form $g(\langle\underline{x}, \underline{\tau}\rangle)$, where the $\mathbb{C}$-valued function $g$ depends on the inner product of the vector variable $\underline{x}$ with a general but fixed vector $\underline{\tau} \in \mathbb{C}^{m}$.

In the case where $g$ is a holomorphic in the complex plane, these plane waves are harmonic if

$$
\Delta_{\underline{x}} g(\langle\underline{x}, \underline{\tau}\rangle)=g^{\prime \prime}(\langle\underline{x}, \underline{\tau}\rangle) \underline{\tau}^{2}=0,
$$

and therefore if $\underline{\tau}$ is an isotropic vector. More specifically, for $\underline{\tau}=\underline{t}+i \underline{s}$ with $\underline{t}, \underline{s} \in \mathbb{R}^{m}$ we need that

$$
\underline{\tau}^{2}=\underline{t}^{2}-\underline{s}^{2}-2 i\langle\underline{t}, \underline{s}\rangle=\|\underline{s}\|^{2}-\|\underline{t}\|^{2}-2 i\langle\underline{t}, \underline{s}\rangle=0,
$$

and hence $\|\underline{s}\|=\|\underline{t}\|$ and $\langle\underline{t}, \underline{s}\rangle=0$. Therefore, we may consider the vectors $\underline{t}, \underline{s}$ to be orthogonal elements of the unit sphere $\mathbb{S}^{m-1}$ of $\mathbb{R}^{m}$. Then the isotropic vector $\underline{\tau}$ is subject to the following properties

$$
\left\{\begin{array}{l}
\underline{\tau} \underline{\tau}^{\dagger} \underline{\tau}=4 \underline{\tau} \\
\underline{\tau}^{2}=\left(\underline{\tau}^{\dagger}\right)^{2}=0 \\
\underline{\tau}^{\dagger} \underline{\tau}+\underline{\tau} \underline{\tau}^{\dagger}=4
\end{array}\right.
$$

where $\underline{\tau}^{\dagger}=-\underline{t}+i \underline{s}$ is the so-called Hermitian conjugated of $\underline{\tau}$.

\subsection{Case $\mathbf{x}=\underline{x}$ and $\mathbf{y}=\underline{y}$.}

In this case, the superdimensions of the vector variables $\mathbf{x}$ and $\mathbf{y}$ are $P=-2 r$ and $Q=q$ respectively, i.e. $p=s=0$.

As seen in the previous section, we have basically two equivalent forms of constructing hypermonogenic functions. We either take $F_{2 r}$ as initial function and proceed as indicated in Proposition 1 or we take $F_{0}$ as initial function and apply Corollary 1 . The aforementioned isotropic vector $\underline{\tau}$ allows to easily construct examples of monogenic plane waves using the first method. Indeed, due to the factorization of the laplacian $\Delta_{\underline{x}}=-\partial_{\underline{x}}^{2}$ one easily obtains, for every holomorphic function $g$, that

$$
\partial_{\underline{x}} g(\langle\underline{x}, \underline{\tau}\rangle)=g^{\prime}(\langle\underline{x}, \underline{\tau}\rangle) \underline{\tau}
$$

is a monogenic plane wave. Hence, our candidate for the initial monogenic plane wave in the hypermonogenic system (9) is

$$
F_{2 r}(\underline{y})=g(\langle\underline{y}, \underline{\tau}\rangle) \underline{\tau} .
$$

The next two elements of this hypermonogenic system have to submit the relations

$$
c(-2 r, 2 r) F_{2 r}(\underline{y})=-\partial_{\underline{y}}\left[F_{2 r-1}(\underline{y})\right] \quad \text { and } \quad c(-2 r, 2 r-1) F_{2 r-1}(\underline{y})=\partial_{\underline{y}}\left[F_{2 r-2}(\underline{y})\right] .
$$

Based on the properties (10), this system may be solved by writing

$$
F_{2 r-1}(\underline{y})=c_{2 r-1} g_{1}(\langle\underline{y}, \underline{\tau}\rangle) \underline{\tau}^{\dagger} \underline{\tau}, \quad F_{2 r-2}(\underline{y})=c_{2 r-2}\left\langle\underline{y}, \underline{\tau}^{\dagger}\right\rangle g_{1}(\langle\underline{y}, \underline{\tau}\rangle) \underline{\tau},
$$

where $g_{1}^{\prime}=g$ and $c_{2 r-1}, c_{2 r-2}$ are suitable constants. Repeating this reasoning for the rest of functions $F_{2 r-3}, \ldots, F_{0}$ we arrive to the system

$$
\begin{aligned}
F_{2 j}(\underline{y}) & =c_{2 j}\left\langle\underline{y}, \underline{\tau}^{\dagger}\right\rangle^{r-j} G^{(j)}(\langle\underline{y}, \underline{\tau}\rangle) \underline{\tau}, & & j=0, \ldots, r, \\
F_{2 j-1}(\underline{y}) & =c_{2 j-1}\left\langle\underline{y}, \underline{\tau}^{\dagger}\right\rangle^{r-j} G^{(j-1)}(\langle\underline{y}, \underline{\tau}\rangle) \underline{\tau}^{\dagger} \underline{\tau}, & j & =1, \ldots, r,
\end{aligned}
$$


where $G: \mathbb{C} \rightarrow \mathbb{C}$ is an arbitrary holomorphic function. Indeed, it is easily seen that this family of functions satisfies

$$
\begin{aligned}
& \partial_{\underline{y}}\left[F_{2 j-1}(\underline{y})\right]=4 c_{2 j-1}\left\langle\underline{y}, \underline{\tau}^{\dagger}\right\rangle^{r-j} G^{(j)}(\langle\underline{y}, \underline{\tau}\rangle) \underline{\tau}, \\
& \partial_{\underline{y}}\left[F_{2 j-2}(\underline{y})\right]=(r-j+1) c_{2 j-2}\left\langle\underline{y}, \underline{\tau}^{\dagger}\right\rangle^{r-j} G^{(j-1)}(\langle\underline{y}, \underline{\tau}\rangle) \underline{\tau}^{\dagger} \underline{\tau} .
\end{aligned}
$$

Hence, the family of plane waves (11) is a solution of the system (9) if the constants $c_{j}$ satisfy the recursion relations

$$
\begin{cases}c_{2 j}=-\frac{2}{j} c_{2 j-1}, & j=1, \ldots, r, \\ c_{2 j-1}=-\frac{1}{2} c_{2 j-2}, & j=1, \ldots, r,\end{cases}
$$

where we are free to choose the value for $c_{0}$. We then set $c_{0}=1$. The above formulas lead to two different recursion formulas for the coefficients $c_{j}$ with even and odd sub-indices respectively

$$
\begin{cases}c_{2 j}=\frac{c_{2 j-2}}{j}, & j=1, \ldots, r, \\ c_{2 j+1}=\frac{c_{2 j-1}}{j}, & j=1, \ldots, r-1,\end{cases}
$$

where $c_{0}=1$ and $c_{1}=-\frac{1}{2}$. Hence, we obtain the explicit expressions

$$
\begin{cases}c_{2 j}=\frac{1}{j !}, & j=0, \ldots, r, \\ c_{2 j-1}=-\frac{1}{2(j-1) !}, & j=1, \ldots, r .\end{cases}
$$

In this way, we have arrived at the hypermonogenic superfunction

$$
\begin{aligned}
F(\underline{x}, \underline{y}) & =\sum_{j=0}^{r} \underline{x}^{2 j} F_{2 j}(\underline{y})+\sum_{j=1}^{r} \underline{x}^{2 j-1} F_{2 j-1}(\underline{y}) \\
& =\sum_{j=0}^{r} \frac{\underline{x}^{2 j}}{j !}\left\langle\underline{y}, \underline{\tau}^{\dagger}\right\rangle^{r-j} G^{(j)}(\langle\underline{y}, \underline{\tau}\rangle) \underline{\tau}-\frac{1}{2} \sum_{j=0}^{r} \frac{\underline{x}^{2 j+1}}{j !}\left\langle\underline{y}, \underline{\tau}^{\dagger}\right\rangle^{r-j-1} G^{(j)}\left(\left\langle\underline{y}, \underline{\tau}^{j}\right\rangle\right) \underline{\tau}^{\dagger} \underline{\tau} \\
& =\left\langle\underline{y}, \underline{\tau}^{\dagger}\right\rangle^{r} \underline{\tau} \sum_{j=0}^{r} \frac{\left(\underline{x^{2}}\left\langle\underline{y}, \underline{\tau}^{\dagger}\right\rangle^{-1}\right)^{j}}{j !} G^{(j)}(\langle\underline{y}, \underline{\tau}\rangle)-\frac{\underline{x}\left\langle\underline{y}, \underline{\tau}^{\dagger}\right\rangle^{r-1}}{2} \underline{\tau}^{\dagger} \underline{\tau} \sum_{j=0}^{r} \frac{\left(\underline{\underline{x}}\left\langle\underline{y}, \underline{\tau}^{\dagger}\right\rangle^{-1}\right)^{j}}{j !} G^{(j)}(\langle\underline{y}, \underline{\tau}\rangle) \\
& =\left(\left\langle\underline{y}, \underline{\tau}^{\dagger}\right\rangle^{r} \underline{\tau}-\frac{\underline{x}\left\langle\underline{y}, \underline{\tau}^{\dagger}\right\rangle^{r-1}}{2} \underline{\tau}^{\dagger} \underline{\tau}\right) G\left(\langle\underline{y}, \underline{\tau}\rangle+\frac{\underline{x^{2}}}{\left\langle\underline{y}, \underline{\tau}^{\dagger}\right\rangle}\right),
\end{aligned}
$$

where $G\left(\langle\underline{y}, \underline{\tau}\rangle+\frac{\underline{x}^{2}}{\left\langle\underline{y}, \underline{\tau}^{\dagger}\right\rangle}\right)$ is defined by its Taylor expansion as in (6).

The same result can be obtained if we apply Corollary 1 with initial function

$$
F_{0}(\underline{y})=\left\langle\underline{y}, \underline{\tau}^{\dagger}\right\rangle^{r} G(\langle\underline{y}, \underline{\tau}\rangle) \underline{\tau} \text {. }
$$

\subsection{Case $\mathrm{x}=\underline{\grave{x}}$ and $\mathrm{y}=\underline{y}$.}

In this case, the superdimensions of the vector variables $\mathbf{x}$ and $\mathbf{y}$ are $P=-2 r$ and $Q=-2 s$ respectively, i.e. $p=q=0$.

In order to construct the first example in this case we consider fermionic plane waves as initial function $F_{0}$, i.e. functions of the form

$$
F_{0}=g\left(\left\langle\underline{y}, \underline{\tau}^{\dagger}\right\rangle\right)=\sum_{j=0}^{2 s} \frac{\left\langle\underline{y}, \underline{\tau}^{\dagger}\right\rangle^{j}}{j !} g^{(j)}(0)
$$


where $g: \mathbb{C} \rightarrow \mathbb{C}$ is analytic in a neighborhood of $z=0$ and $\underline{\underline{\imath}}=\sum_{j=1}^{2 s} \grave{\tau}_{j} \grave{e_{2 r+j}}$ is a fermionic vector parameter.

Formula (12) shows that it only suffices to consider functions of the form $F_{0}(\underline{y})=\langle\underline{y}, \underline{\tau}\rangle^{j}$ with $0 \leq j \leq 2 s$. These functions must satisfy the condition $\partial_{\underline{y}}^{2 r+1} F_{0}=0$, which is always true if $s \leq r$. Let us consider, as an illustrating example, the limit case in which $s=r$ and $F_{0}(\underline{y})=\langle\underline{y}, \underline{\tau}\rangle^{2 r}$. Other interesting options shall be considered in future work.

One has the following identities for this choice

$$
\partial_{\underline{\underline{y}}}^{j} F_{0}=(-1)^{j} \frac{(2 r) !}{(2 r-j) !} \underline{\tau}^{j}\langle\underline{y}, \underline{\tau}\rangle^{2 r-j}, \quad j=0,1, \ldots, 2 r .
$$

Then by Corollary 1 one has that

$$
\begin{aligned}
& F(\underline{\grave{x}}, \underline{\grave{y}})=\frac{(2 r) !}{r !}\left(\sum_{j=0}^{r} \frac{(r-j) !}{j !(2 r-2 j) !} \frac{\underline{x}^{2 j} \underline{\underline{\tau}}^{2 j}}{2^{2 j}}\langle\underline{\grave{y}}, \underline{\tau}\rangle^{2 r-2 j}-\frac{\underline{x}^{\grave{\tau}}}{2} \sum_{j=0}^{r-1} \frac{(r-j-1) !}{j !(2 r-2 j-1) !} \frac{\underline{x}^{2 j} \underline{\underline{\tau}}^{2 j}}{2^{2 j}}\left\langle\underline{\grave{y}}, \underline{\tau}^{\dagger}\right\rangle^{2 r-2 j-1}\right) \\
& =\frac{(2 r) !}{r !}\left\langle\underline{\grave{y}}, \underline{\tau}^{\prime}\right\rangle^{2 r-1}\left(\langle\underline{\underline{y}}, \underline{\tau}\rangle \sum_{j=0}^{r} \frac{(r-j) !}{j !(2 r-2 j) !} z^{j}-\frac{\grave{x} \underline{\underline{\tau}}}{2} \sum_{j=0}^{r-1} \frac{(r-j-1) !}{j !(2 r-2 j-1) !} z^{j}\right)
\end{aligned}
$$

where we have formally written $z=\frac{\underline{x}^{2} \underline{\tau}^{2}}{4\langle\underline{y}, \underline{\tau}\rangle^{2}}$. Here the inverse notation for the nilpotent element $\langle\underline{y}, \underline{\tau}\rangle$ is purely formal, only positive powers of this element will occur in the resulting sum after the multiplication by $\langle\underline{\hat{y}}, \underline{\underline{\tau}}\rangle^{2 r}$.

It is easily seen that

$$
\sum_{j=0}^{r} \frac{(r-j) !}{j !(2 r-2 j) !} z^{j}=\frac{z^{r}}{r !}{ }_{2} F_{1}\left(1,-r ; \frac{1}{2} ; \frac{-1}{4 z}\right)
$$

and

$$
\sum_{j=0}^{r-1} \frac{(r-j-1) !}{j !(2 r-2 j-1) !} z^{j}=\frac{z^{r-1}}{(r-1) !}{ }_{2} F_{1}\left(1,1-r ; \frac{3}{2} ; \frac{-1}{4 z}\right),
$$

where ${ }_{2} F_{1}(a, b ; c ; z)$ denotes the hypergeometric function, see [1, p. 64].

Finally, we can formally write

$$
\begin{aligned}
& F(\underline{\grave{x}}, \underline{y})=\frac{(2 r) !}{r !(r-1) !}\langle\underline{\grave{y}}, \underline{\underline{\gamma}}\rangle^{2 r-1} z^{r-1}\left[\frac{\langle\underline{\grave{y}}, \underline{\underline{\tau}}\rangle z}{r}{ }_{2} F_{1}\left(1,-r ; \frac{1}{2} ; \frac{-1}{4 z}\right)-\frac{\grave{x} \grave{\underline{\tau}}}{2}{ }_{2} F_{1}\left(1,1-r ; \frac{3}{2} ; \frac{-1}{4 z}\right)\right] \\
& =\frac{(2 r) !}{r !(r-1) !}\left[\frac{\underline{x}^{2 r} \underline{\tau}^{2 r}}{2^{2 r} r}{ }_{2} F_{1}\left(1,-r ; \frac{1}{2} ; \frac{-\langle\underline{y}, \underline{\underline{\tau}}\rangle^{2}}{\underline{\underline{x}}^{2} \underline{\underline{\tau}}^{2}}\right)-\frac{\langle\underline{y}, \underline{\underline{\tau}}\rangle \underline{x}^{2 r-1} \underline{\underline{\tau}}^{2 r-1}}{2^{2 r-1}}{ }_{2} F_{1}\left(1,1-r ; \frac{3}{2} ; \frac{-\langle\underline{y}, \underline{\underline{\tau}}\rangle^{2}}{\underline{\underline{x}}^{2} \underline{\tau}^{2}}\right)\right] .
\end{aligned}
$$

We recall that the inverse notation for the nilpotent element $\underline{x}^{2} \underline{\tau}^{2}$ is purely formal, only positive powers of this element will occur in the resulting sum after the multiplication by $\underline{x}^{2 r} \underline{\tau}^{2 r}$.

A second example of hypermonogenic plane wave can be constructed by choosing the fermionic vector parameter $\underline{\tau}$ to be isotropic. Indeed, let us consider a vector parameter

$$
\underline{t}=\sum_{j=1}^{s}{\grave{t_{2 j-1}}}_{2 r+2 j-1}+\grave{t}_{2 j} e_{2 r+2 j}
$$


and the action of the complex structure $J=\operatorname{diag}\left(\begin{array}{cc}0 & 1 \\ -1 & 0\end{array}\right) \in \operatorname{Sp}(2 n)$ on it, see [13],

$$
J(\underline{\underline{t}})=\sum_{j=1}^{s} \grave{t}_{2 j} e_{2 r+2 j-1}-\grave{t}_{2 j-1} e_{2 r+2 j} \text {. }
$$

We then have that

$$
\underline{t}^{2}=2 \sum_{j=1}^{s} \grave{t}_{2 j-1} \hat{t}_{2 j}=J\left(\underline{t^{\prime}}\right)^{2} \quad \text { and } \quad \underline{t} J(\underline{t})+J(\underline{t}) \underline{t}=\sum_{j=1}^{s} \grave{t}_{2 j-1}^{2}+t_{2 j}^{2}=0 .
$$

Hence if we write

$$
\underline{\grave{\imath}}=\underline{\grave{t}}+i J(\underline{\hat{t}}), \quad \underline{\underline{\tau}}^{\dagger}=-\underline{t}+i J(\underline{\hat{t}}),
$$

we get, in a similar way to (10), the following identities

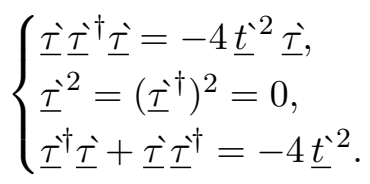

The same set of identities can be obtained if, instead of the complex structure $J$, one uses any other superrotation $M$ that orthogonally rotates the supervector $\underline{t}$, i.e. a linear operator $M$ satisfying $\underline{t} M(\underline{t})+M(\underline{t}) \underline{t}=0$. For an introduction to superrotations and their use in Clifford analysis we refer the reader to [12] and [14].

We can now proceed, as in the previous case 4.1, by considering the initial function

$$
F_{0}(\underline{y})=\left\langle\underline{y}, \underline{\tau}^{\dagger}\right\rangle^{r}\left\langle\underline{y}, \underline{\tau}^{\dagger}\right\rangle^{r} \underline{\tau} .
$$

Recall that $\partial_{\underline{y}}\left\langle\underline{y}, \underline{\tau}^{\dagger}\right\rangle=-\frac{1}{2} \partial_{\underline{y}}\left\{\underline{y}, \underline{\tau}^{\dagger}\right\}=-\underline{\tau}^{\dagger}$, which implies $\partial_{\underline{y}}\left\langle\underline{y}, \underline{\tau}^{\dagger}\right\rangle^{j}=-j\left\langle\underline{y}, \underline{\tau}^{\dagger}\right\rangle^{j-1} \underline{\tau}^{\dagger}$. Thus we have that

$$
\begin{aligned}
\partial_{\underline{y}}^{2 j} F_{0} & =(-1)^{j} 2^{2 j} \underline{t}^{2 j} \frac{r !^{2}}{(r-j) !^{2}}\left\langle\underline{y}, \underline{\tau}^{\dagger}\right\rangle^{r-j}\langle\underline{y}, \underline{\underline{\tau}}\rangle^{r-j} \underline{\underline{\tau}}, & j=0,1, \ldots, r, \\
\partial_{\underline{\underline{y}}}^{2 j+1} F_{0} & =(-1)^{j+1} 2^{2 j} \underline{t}^{2 j} \frac{r !^{2}}{(r-j) !(r-j-1) !}\left\langle\underline{y}, \underline{\tau}^{\dagger}\right\rangle^{r-j-1}\left\langle\underline{y}, \underline{\tau}^{\dagger}\right\rangle^{r-j} \underline{\tau}^{\dagger} \underline{\tau}, & j=0,1, \ldots, r-1 .
\end{aligned}
$$

Observe that for $j=r$ we obtain the constant

$$
\partial_{\underline{\underline{y}}}^{2 r} F_{0}=(-1)^{r} 2^{2 r} \underline{t}^{2 r} r !^{2} \underline{\underline{\tau}}
$$

which implies that $\partial_{\underline{\underline{y}}}^{2 r+1} F_{0}=0$. Applying then Corollary 1 one has

$$
\begin{aligned}
& F(\underline{x}, \underline{y})=r !\left(\sum_{j=0}^{r} \frac{(-1)^{j} \underline{t}^{2 j} \underline{x}^{2 j}\left\langle\underline{\grave{y}}, \underline{\tau}^{\dagger}\right\rangle^{r-j}\langle\underline{y}, \underline{\underline{\tau}}\rangle^{r-j}}{j !(r-j) !}\right) \underline{\tau} \\
& +r ! \frac{\underline{x}\langle\underline{y}, \underline{\tau}\rangle}{2}\left(\sum_{j=0}^{r-1} \frac{(-1)^{j+1} \underline{\hat{t}}^{2 j} \underline{x}^{2 j}\left\langle\underline{y}, \underline{\tau}^{\dagger}\right\rangle^{r-j-1}\langle\underline{y}, \underline{\tau}\rangle^{r-j-1}}{j !(r-j) !}\right) \underline{\tau}^{\dagger} \underline{\underline{\tau}} \\
& =\left(\left\langle\underline{y}, \underline{\tau}^{\dagger}\right\rangle\langle\underline{y}, \underline{\tau}\rangle-\underline{t}^{2} \underline{x}^{2}\right)^{r} \grave{\underline{\tau}}-\frac{\grave{x}}{2\left\langle\underline{y}, \underline{\tau}^{\dagger}\right\rangle}\left(\left\langle\underline{y}, \underline{\tau}^{\dagger}\right\rangle\langle\underline{y}, \underline{\tau}\rangle-\underline{t}^{2} \underline{x}^{2}\right)^{r} \underline{\underline{\tau}}^{\dagger} \underline{\tau}^{\prime} \\
& =\left(\grave{\tau}-\frac{\grave{x}}{2\left\langle\underline{y}, \underline{\tau}^{\dagger}\right\rangle} \underline{\tau}^{\dagger}{ }^{\dagger}\right)\left(\left\langle\underline{y}, \underline{\tau}^{\dagger}\right\rangle\langle\underline{y}, \underline{\tau}\rangle-\underline{t}^{2} \underline{x}^{2}\right)^{r} .
\end{aligned}
$$

The inverse notation for the nilpotent element $\left\langle\underline{y}, \underline{\tau}^{\dagger}\right\rangle$ in the last two equalities is purely formal, only positive powers of this element will occur in the resulting sum. 


\subsection{Case $\mathbf{x}=\underline{x}$ and $\mathbf{y}=\underline{y}$.}

In this case, the superdimensions of the vector variables $\mathbf{x}$ and $\mathbf{y}$ are $P=p$ and $Q=-2 s$ respectively, i.e. $r=q=0$.

By Proposition 2 we have that any hypermonogenic function in this case has the form

$$
F(\underline{x}, \underline{y})=\Gamma\left(\frac{p}{2}\right)\left(\frac{|\underline{x}| \sqrt{\Delta_{\underline{y}}}}{2}\right)^{-\frac{p}{2}}\left(\frac{|\underline{x}| \sqrt{\Delta_{\underline{y}}}}{2} J_{\frac{p}{2}-1}\left(|\underline{x}| \sqrt{\Delta_{\underline{y}}}\right)-\frac{\underline{x} \partial_{\underline{y}}}{2} J_{\frac{p}{2}}\left(|\underline{x}| \sqrt{\Delta_{\underline{y}}}\right)\right)\left[F_{0}\right] .
$$

Since the operator $|\underline{x}| \sqrt{\Delta_{\underline{y}}}$ is nilpotent, we have that

$$
F(\underline{x}, \underline{y})=\Gamma\left(\frac{p}{2}\right)\left(\sum_{j=0}^{s} \frac{(-1)^{j}|\underline{x}|^{2 j}}{2^{2 j} j ! \Gamma\left(\frac{p}{2}+j\right)} \Delta_{\underline{y}}^{j} F_{0}-\frac{\underline{x} \partial_{\underline{y}}}{2} \sum_{j=0}^{s-1} \frac{(-1)^{j}|\underline{x}|^{2 j}}{2^{2 j} j ! \Gamma\left(\frac{p}{2}+j+1\right)} \Delta_{\underline{y}}^{j} F_{0}\right) .
$$

Thus the series expansion (8) of the function $F(\underline{x}, \underline{y})$ still has a finite number of elements as in the previous cases.

Formula (12) shows that the powers $\left\langle\grave{y}, \underline{\tau}^{j}\right\rangle^{j}, j=0,1, \ldots, 2 s$, constitute a basis for the space of all plane waves depending only on the inner product $\langle\grave{y}, \underline{\tau}\rangle$. To construct our example in this case, we consider the biggest of these powers as initial function, i.e. $F_{0}(\underline{y})=\langle\underline{y}, \underline{\tau}\rangle^{2 s}$.

We recall that

$$
\begin{array}{cl}
(-1)^{j} \Delta_{\underline{y}}^{j} F_{0}=\partial_{\underline{y}}^{2 j} F_{0}=\frac{(2 s) !}{(2 s-2 j) !} \underline{\tau}^{2 j}\left\langle\underline{y}, \underline{\tau}^{\dagger}\right\rangle^{2 s-2 j}, & j=0, \ldots, s, \\
(-1)^{j} \partial_{\underline{y}} \Delta_{\underline{y}}^{j} F_{0}=\partial_{\underline{\underline{y}}}^{2 j+1} F_{0}=\frac{-(2 s) !}{(2 s-2 j-1) !} \underline{\tau}^{2 j+1}\langle\underline{y}, \underline{\tau}\rangle^{2 s-2 j-1}, & j=0, \ldots, s-1 .
\end{array}
$$

Hence,

$$
F(\underline{x}, \underline{y})=\Gamma\left(\frac{p}{2}\right)(2 s) !\left(\sum_{j=0}^{s} \frac{|\underline{x}|^{2 j} \underline{\underline{\tau}}^{2 j}\langle\underline{y}, \underline{\tau}\rangle^{2 s-2 j}}{2^{2 j} j ! \Gamma\left(\frac{p}{2}+j\right)(2 s-2 j) !}+\frac{\underline{x} \underline{\underline{\tau}}}{2} \sum_{j=0}^{s-1} \frac{|\underline{x}|^{2 j} \underline{\tau}^{2 j}\langle\underline{y}, \underline{\tau}\rangle^{2 s-2 j-1}}{2^{2 j} j ! \Gamma\left(\frac{p}{2}+j+1\right)(2 s-2 j-1) !}\right) .
$$

Observe now that the following identities hold,

$$
\sum_{j=0}^{s} \frac{a^{2 j} b^{2 s-2 j}}{2^{2 j} j ! \Gamma\left(\frac{p}{2}+j\right)(2 s-2 j) !}=\frac{b^{2 s}{ }_{2} F_{1}\left(-s,-s+\frac{1}{2} ; \frac{p}{2} ; \frac{a^{2}}{b^{2}}\right)}{\Gamma\left(\frac{p}{2}\right)(2 s) !}
$$

and

$$
\sum_{j=0}^{s-1} \frac{a^{2 j} b^{2 s-2 j-1}}{2^{2 j} j ! \Gamma\left(\frac{p}{2}+j+1\right)(2 s-2 j-1) !}=\frac{b^{2 s-1}{ }_{2} F_{1}\left(-s+1,-s+\frac{1}{2} ; \frac{p}{2}+1 ; \frac{a^{2}}{b^{2}}\right)}{\Gamma\left(\frac{p}{2}+1\right)(2 s-1) !} .
$$

Then if we write $a=|\underline{x}| \underline{\underline{\tau}}$ and $b=\langle\underline{y}, \underline{\tau}\rangle$ we get

$$
F(\underline{x}, \underline{y})=\langle\underline{y}, \underline{\tau}\rangle^{2 s}{ }_{2} F_{1}\left(-s,-s+\frac{1}{2} ; \frac{p}{2} ; \frac{\underline{x}^{2} \underline{\tau}^{2}}{\langle\underline{y}, \underline{\tau}\rangle^{2}}\right)+\frac{2 s}{p} \underline{x} \underline{\tau}\langle\underline{y}, \underline{\tau}\rangle^{2 s-1}{ }_{2} F_{1}\left(-s+1,-s+\frac{1}{2} ; \frac{p}{2}+1 ; \frac{\underline{x}^{2} \underline{\tau}^{2}}{\langle\underline{y}, \underline{\tau}\rangle^{2}}\right),
$$

where the inverse notation for the nilpotent element $\langle\underline{y}, \underline{\tau}\rangle$ is purely formal, only positive powers of this element will occur in the resulting sum. 


\section{Concluding remarks}

In this paper, we have studied some particular cases of hypermonogenic superfunctions considering only purely bosonic and fermionic vector variables. The use of this cases has lead to considerable different features with respect to the classical setting in which only a pair of bosonic vector variables are considered, see [15]. In future work, we shall address this study for a pair of supervector variables with arbitrary superdimensions $P, Q \in \mathbb{Z}$. 


\begin{tabular}{|c|c|c|}
\hline $\begin{array}{c}\mathbf{x}=\underline{x}, \mathbf{y}=\underline{y} \\
P=-2 r, Q=q\end{array}$ & $\begin{aligned} F_{2 r}(\underline{y}) & =\frac{1}{r !} G^{(r)}(\langle\underline{y}, \underline{\tau}\rangle) \underline{\tau} \\
\text { or } F_{0}(\underline{y}) & =\left\langle\underline{y}, \underline{\tau}^{\dagger}\right\rangle^{r} G(\langle\underline{y}, \underline{\tau}\rangle) \underline{\tau}\end{aligned}$ & $\left(\left\langle\underline{y}, \underline{\tau}^{\dagger}\right\rangle^{r} \underline{\tau}-\frac{\underline{x}\left\langle\underline{y}, \underline{\tau}^{\dagger}\right\rangle^{r-1}}{2} \underline{\tau}^{\dagger} \underline{\tau}\right) G\left(\langle\underline{y}, \underline{\tau}\rangle+\frac{\grave{x}^{2}}{\left\langle\underline{y}, \underline{\tau}^{\dagger}\right\rangle}\right)$ \\
\hline $\begin{array}{c}\mathbf{x}=\underline{x}, \mathbf{y}=\underline{y} \\
P=-2 r, Q=-2 s\end{array}$ & $\begin{array}{l}F_{0}(\underline{y})=\left\langle\underline{y}, \underline{\tau}^{2 r}(s=r)\right. \\
F_{0}(\underline{y})=\left\langle\underline{y}, \underline{\tau}^{\dagger}\right\rangle^{r}\langle\underline{y}, \underline{\tau}\rangle^{r} \underline{\tau}\end{array}$ & 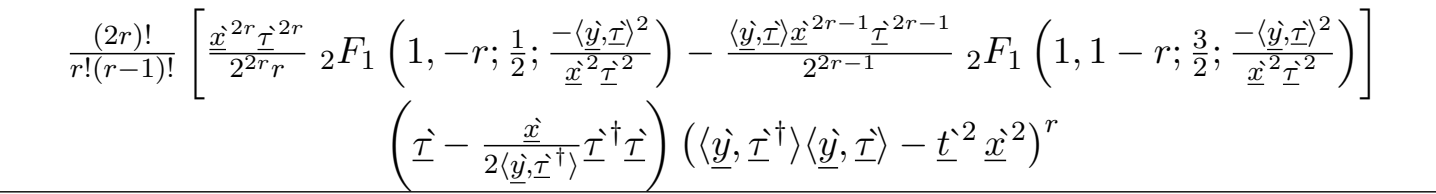 \\
\hline
\end{tabular}

Table 1: Examples of hypermonogenic plane waves in superspace. 


\section{References}

[1] G. E. Andrews, R. Askey, and R. Roy. Special functions, volume 71 of Encyclopedia of Mathematics and its Applications. Cambridge University Press, Cambridge, 1999.

[2] F. A. Berezin. Introduction to superanalysis, volume 9 of Mathematical Physics and Applied Mathematics. D. Reidel Publishing Co., Dordrecht, 1987. Edited and with a foreword by A. A. Kirillov, With an appendix by V. I. Ogievetsky, Translated from the Russian by J. Niederle and R. Kotecký, Translation edited by Dimitri Leŭtes.

[3] F. Brackx, R. Delanghe, and F. Sommen. Clifford analysis, volume 76 of Research Notes in Mathematics. Pitman (Advanced Publishing Program), Boston, MA, 1982.

[4] K. Coulembier and H. De Bie. Conformal symmetries of the super Dirac operator. Rev. Mat. Iberoam., 31(2):373-410, 2015.

[5] H. De Bie, D. Eelbode, and F. Sommen. Spherical harmonics and integration in superspace. II. J. Phys. A, 42(24):245204, 18, 2009.

[6] H. De Bie and F. Sommen. A Clifford analysis approach to superspace. Ann. Physics, 322(12):2978-2993, 2007.

[7] H. De Bie and F. Sommen. Correct rules for Clifford calculus on superspace. Adv. Appl. Clifford Algebr., 17(3):357-382, 2007.

[8] H. De Bie and F. Sommen. Spherical harmonics and integration in superspace. J. Phys. A, 40(26):7193-7212, 2007.

[9] H. De Bie and F. Sommen. Fischer decompositions in superspace. In Function spaces in complex and Clifford analysis, pages 170-188. Natl. Univ. Publ. Hanoi, Hanoi, 2008.

[10] H. De Bie and F. Sommen. Fundamental solutions for the super Laplace and Dirac operators and all their natural powers. J. Math. Anal. Appl., 338(2):1320-1328, 2008.

[11] H. De Bie and F. Sommen. A Cauchy integral formula in superspace. Bull. Lond. Math. Soc., 41(4):709-722, 2009.

[12] H. De Schepper, A. Guzmán Adán, and F. Sommen. The spin group in superspace. Submitted for publication, arXiv:1804.00963 [math.GR].

[13] H. De Schepper, A. Guzmán Adán, and F. Sommen. Hermitian Clifford Analysis on Superspace. Adv. Appl. Clifford Algebr., 28(1):28:5, 2018.

[14] H. De Schepper, A. Guzmán Adán, and F. Sommen. Spin actions in Euclidean and Hermitian Clifford analysis in superspace. J. Math. Anal. Appl., 457(1):23-50, 2018.

[15] R. Delanghe, F. Sommen, and V. Souček. Clifford algebra and spinor-valued functions, volume 53 of Mathematics and its Applications. Kluwer Academic Publishers Group, Dordrecht, 1992. A function theory for the Dirac operator, Related REDUCE software by F. Brackx and D. Constales, With 1 IBM-PC floppy disk (3.5 inch).

[16] B. DeWitt. Supermanifolds. Cambridge Monographs on Mathematical Physics. Cambridge University Press, Cambridge, 1984. 
[17] S.-L. Eriksson and H. Leutwiler. Introduction to hyperbolic function theory. Clifford Algebras and Inverse Problems, Tampere Univ. of Tech. Institute of Math. Research Report No. 90 (2009):1-28, 2009.

[18] S.-L. Eriksson, H. Orelma, and F. Sommen. Vekua systems in hyperbolic harmonic analysis. Complex Anal. Oper. Theory, 10(2):251-265, 2016.

[19] S.-L. Eriksson, H. Orelma, and N. Vieira. Hypermonogenic functions of two vector variables. Complex Anal. Oper. Theory, 12(2):555-570, 2018.

[20] J. E. Gilbert and M. A. M. Murray. Clifford algebras and Dirac operators in harmonic analysis, volume 26 of Cambridge Studies in Advanced Mathematics. Cambridge University Press, Cambridge, 1991.

[21] A. Guzmán Adán. Euclidean and Hermitian Clifford analysis on superspace. PhD thesis, Ghent University, 2018.

[22] A. Guzmán Adán, H. Orelma, and F. Sommen. Hypermonogenic solutions and plane waves of the Dirac operator in $\mathbb{R}^{p} \times \mathbb{R}^{q}$. Appl. Math. Comput., 346:1-14, 2019.

[23] A. Guzmán Adán and F. Sommen. Distributions and integration in superspace. J. Math. Phys., 59(7):073507, 25, 2018.

[24] D. A. Leites. Introduction to the theory of supermanifolds. Uspekhi Mat. Nauk, 35(1(211)):3$57,255,1980$.

[25] H. Leutwiler. Modified Clifford analysis. Complex Variables Theory Appl., 17(3-4):153-171, 1992. 